\title{
Stress ocupacional em bombeiros: efeitos de intervenção baseada em avaliação de necessidades ${ }^{1}$
}

\section{Firefighters' occupational stress: intervention effects based on needs assessment}

\author{
Sheila Giardini MURTA ${ }^{2}$ \\ Bartholomeu Tôrres TRÓCCOLl ${ }^{3}$
}

\begin{abstract}
Resumo
Este estudo descreve os efeitos de uma intervenção para o manejo do stress ocupacional, baseada em avaliação de necessidades junto a sete bombeiros. Entrevistas grupais foram feitas para identificar estressores ocupacionais, estratégias de coping e sintomas de stress. Baseando-se em evidências de que a população-alvo apresentava um repertório restrito de coping e muitos sintomas de stress, implementou-se uma intervenção com 12 sessões, contendo informação, relaxamento, treino assertivo, treino em solução de problemas, manejo de tempo e reestruturação cognitiva. Medidas pré e pós-teste foram obtidas para imunidade, pressão arterial e respostas verbais de stress, saúde geral e coping. A comparação entre medidas pré e pós-intervenção evidenciou redução em stress e sintomas somáticos; aumento em auto-eficácia e em pressão arterial diastólica. Intervenções organizacionais ou combinadas foram recomendadas.
\end{abstract}

Unitermos: avaliação de necessidades; intervenção psicológica; stress ocupacional.

\begin{abstract}
This study describes effects of an occupational stress intervention based on needs assessment in seven firefighters. Group interviews were conducted to identify occupational stressors, coping strategies and stress symptoms. Based on evidences that the target-group presented a restrict coping repertoire and many stress symptoms, it was implemented a 12-sessions intervention, consisted by information, relaxation, assertive training, problem solution skills, time management and cognitive restructuring. Pre and post-test measures were obtained for immunity, blood pressure, and verbal responses of stress, general health and coping. The comparison of pre and post-measures showed stress and somatic symptoms decrease; and self-efficacy and diastolic blood pressure increase. Organizational or combined interventions were recommended.
\end{abstract}

Uniterms: needs assessment; psychological intervention; occupational stress.

$\boldsymbol{\nabla \nabla \nabla \nabla}$

1 Artigo elaborado a partir da tese de S.G. MURTA, intitulada: "Planejamento, implementação e avaliação de um Programa de manejo de stress ocupacional". Universidade de Brasília, 2005.

2 Universidade Católica de Goiás, Departamento de Psicologia. Av. Universitária, 1440, Setor Universitário, 74605-010, Goiânia, GO, Brasil. Correspondência para/Correspondence to: S.G. MURTA. E-mail: <murta@cultura.com.br>.

3 Universidade de Brasília, Instituto de Psicologia, Departamento de Psicologia Social e do Trabalho. Brasília DF, Brasil. 
Bombeiros e outros profissionais que lidam com situações de emergência em saúde estão mais susceptíveis ao desenvolvimento de stress no trabalho. Um estudo descritivo (Outtlinger, 1998) feito com 161 bombeiros americanos acerca de fontes de stress vivenciadas no trabalho identificou, como principais fontes estressoras, a morte ou o acidente com colegas de trabalho em serviço, a prestação de ajuda a pessoas jovens seriamente feridas ou mortas e o enfrentamento de problemas sobre os quais não se tem controle e que continuam existindo sem que providências sejam tomadas por outros para minimizá-los.

Além da exposição a riscos psicossociais, os bombeiros lidam também com riscos biológicos, como exposição a sangue contaminado e privação de sono por escala noturna de trabalho ou ciclos longos de trabalho-descanso. Há consenso na literatura de que a privação de sono está entre os estressores associados à diminuição de células do sistema de defesa imunológica (Cohen \& Herbert, 1996; Kiecolt-Glaser, 1999; O'Leary, 1990), o que permite supor que esse grupo ocupacional seja particularmente susceptível a problemas em imunidade e conseqüente vulnerabilidade a doenças diversas.

Adicionalmente, há evidências de que bombeiros são particularmente expostos a fatores de risco para o desenvolvimento de doenças cardíacas, stress pós-traumático e burnout (Corneil, 1995; Harris, Baloglu \&Stacks, 2002; Haslam \& Mallon, 2003; Regehr, Hill, Knott \& Sault, 2003).

O alto risco de stress que os bombeiros estão expostos demonstra que fazem parte de um grupo ocupacional que necessita de programas de manejo de stress ocupacional. Tais programas variam enormemente quanto ao foco, incluindo intervenções focadas no indivíduo (como intervenções baseadas em habilidades comportamentais e cognitivas de coping, meditação, educação em saúde e atividade física), na relação indivíduo-organização (como ações para melhoria da comunicação e trabalho em equipe), na organização (como treinamento e mudança das condições físicoambientais) e intervenções combinadas (conjugação de dois ou mais tipos de intervenção com focos distintos) (van der Heck \& Plomp, 1997).

Intervenções focadas em coping individual são, notadamente, as mais freqüentes na área (van der Klink,

42 Blonk, Schene \& van Dijk, 2001) provavelmente por terem um custo menor com planejamento e implementação, por serem menos intrusivas na rotina organizacional e por não entrarem em confronto direto com práticas administrativas da organização e resistências à mudança (Bellarosa \& Chen, 1997).

Adicionalmente, evidências acumuladas de que o uso de estratégias saudáveis de coping possa favorecer comportamentos de saúde, estados emocionais positivos, relações interpessoais gratificantes e melhor resposta imune (Salovey, Rothman, Detweiler \& Steward, 2000) têm estimulado o desenvolvimento de programas com esse foco.

Há muito se discute que tipo de intervenção nessa área é mais efetivo (Newman \& Beeher, 1979). Até meados dos anos 1990, achados inconclusivos foram encontrados devido aos problemas metodológicos existentes no campo, como ausência de controles e de follow-up. Recentemente, uma metanálise investigou a efetividade dos diferentes tipos de intervenções em manejo de stress ocupacional, utilizando dados de 48 estudos experimentais (van der Klink et al., 2001). Os resultados mostraram que intervenções multimodais com foco individual (junção de técnicas cognitivo-comportamentais e de relaxamento) e cognitivo-comportamentais são mais efetivas em comparação a intervenções focadas em relaxamento e intervenções organizacionais.

Esses autores salientam, todavia, que essas intervenções se mostraram mais efetivas em ambientes organizacionais que permitiam algum controle e decisão por parte do trabalhador. Esses contextos permitiriam maior escolha quanto ao uso e à variação em estratégias de coping.

Já em contextos com baixo controle e decisão por parte do trabalhador, intervenções organizacionais seriam as mais indicadas, juntamente com intervenções cognitivo-comportamentais. Por conseqüência, a inserção de um diagnóstico sobre os estressores ocupacionais e seus efeitos antes da intervenção parece um procedimento indispensável para o sucesso do programa, pois indicaria os focos sobre os quais se deveria intervir (Hawe, Degeling \& Hall, 1990).

Embora tão expostos a fontes de stress, surpreendentemente, os bombeiros raramente têm sido inseridos em programas de manejo de stress rigorosamente avaliados (Murphy, 1996). 
Um dos poucos estudos feitos nesse sentido foi desenvolvido por Calais (2004), que avaliou os efeitos de uma intervenção clínica para tratamento de stress pós-traumático em bombeiros de uma cidade do interior de São Paulo. Participaram 45 bombeiros, divididos em três condições: grupo experimental $(n=15)$, grupo-controle $(n=15)$ e grupo sem tratamento $(n=15)$. 0 grupo experimental participou de oito sessões, de duas horas cada uma, com técnicas cognitivo-comportamentais para controle de stress pós-traumático. O grupo-controle participou de oito palestras dadas por profissionais não psicólogos sobre a prevenção de doenças diversas; e o grupo sem tratamento não participou de qualquer intervenção. Todos os participantes responderam à Escala de Sintomas de Stress Pós-Traumático (Calais, 2004) antes e após a intervenção. Os resultados apontaram diminuição em sintomas de stress pós-traumático nos grupos experimental e sem tratamento e manutenção de sintomas no grupo-controle. A adoção de indicadores conjugados para avaliação de resultados em intervenções futuras foi recomendada por Calais (2004).

Grande parte dos estudos na área se baseia em medidas verbais, como escalas e questionários usados para avaliar sintomas de stress, depressão, ansiedade, assertividade, queixas em saúde e coping, algumas das variáveis dependentes mais investigadas nesse campo (van der Heck \& Plomp, 1997).

Sabe-se, contudo, que como qualquer outra estratégia de coleta de dados usada isoladamente, as escalas e os questionários também têm limitações, sendo particularmente susceptíveis aos efeitos da desejabilidade social (o respondente tende a dar a resposta que seria socialmente aprovada) e distorções de memória (por exemplo, o respondente pode não se lembrar com exatidão do seu real desempenho em uma dada situação).

Como resultado dos problemas nas medidas, as proporções do fenômeno avaliado podem não ser adequadamente acessadas e descritas. Esses problemas podem ser amenizados se aos indicadores verbais forem associados outros tipos de indicadores, como fisiológicos, observacionais e organizacionais, de modo a se obter um panorama mais amplo do objeto em questão.

Numa tentativa de contribuir para suprir as lacunas relativas à avaliação de necessidades e à combiação de diferentes indicadores, o presente estudo implementou e avaliou efeitos de um programa de manejo de stress junto a bombeiros, baseado em avaliação de necessidades prévia, e no uso de indicadores verbais e fisiológicos. Tratou-se de um programa com formato grupal e psicoeducativo - por centrar no fornecimento de informações e estímulo à aprendizagem de novas habilidades de enfrentamento (Afonso et al., 2003) -, do tipo multimodal, por incluir técnicas cognitivo-comportamentais e de relaxamento.

Os objetivos do estudo foram: (a) implementar um programa de manejo de stress ocupacional junto a bombeiros; (b) descrever fontes de stress, estratégias de coping e impacto dos estressores sobre a saúde e; (c) identificar efeitos da intervenção comparando-se medidas pré e pós-intervenção das variáveis sintomas de stress, problemas em saúde geral, coping a problemas no trabalho, pressão arterial sistólica e diastólica e medidas de imunidade (linfócitos, neutrófilos e monócitos). Os linfócitos são células responsáveis pela produção de anticorpos e destruição de células cancerígenas e os macrófagos e neutrófilos são células que fazem fagocitose de microorganismos nocivos e invasores (O'Leary, 1990).

\section{Método}

\section{Participantes}

Participaram do estudo 22 bombeiros de um grupamento de resgate pré-hospitalar (GRPH), do Corpo de Bombeiros do Estado de Goiás. Desses, 15 participaram da primeira fase, destinada ao levantamento de necessidades, e sete participaram da intervenção propriamente dita. Os 15 participantes da fase de levantamento de necessidades eram de ambos os sexos, com tempo na instituição variando entre um mês e oito anos. Todos eram motoristas ou socorristas, isto é, tinham por tarefa atender a indivíduos vítimas de acidentes automobilísticos ou vítimas de outras condições clínicas que necessitavam de socorro hospitalar emergencial.

Os sete bombeiros participantes da intervenção foram voluntários. Cinco deles eram soldados, um era cabo e um outro sargento. Todos exerciam a função de motoristas ou socorristas. O tempo na função variava entre um mês e três anos e trabalhavam em média 67 
horas semanais. Vários deles exerciam atividades profissionais outras que não a de bombeiro, em seus dias de folga. Seis eram do sexo masculino e um do sexo feminino, com idade variando entre 22 e 35 anos e instrução entre $2^{\circ}$ grau e $3^{\circ}$ grau incompleto. Todos os participantes cumpriam uma escala de trabalho de 24 horas consecutivas seguidas por 48 de descanso, sem horário fixo para as refeições durante o período de trabalho. Por força da ocupação, lidavam constantemente com situações imprevisíveis e eram exigidos a prestar atendimento com rapidez.

\section{Avaliação de necessidades}

O programa foi antecedido pela avaliação de necessidades, conduzida por meio de entrevistas grupais não estruturadas, em três grupos de cinco participantes cada um. O primeiro grupo entrevistado foi composto por bombeiros do sexo masculino e novatos no trabalho (até dois anos); o segundo grupo por bombeiros do sexo feminino e o terceiro por bombeiros homens veteranos no trabalho (entre dois e oito anos de trabalho).

Foram coletadas informações acerca das fontes de stress percebidas no trabalho, seu impacto sobre a saúde e estratégias de coping usadas diante dos estressores ocupacionais, cujo registro foi feito por escrito ao longo da entrevista. Cada entrevista teve a duração aproximada de 120 minutos.

\section{Recrutamento}

Finalizada a avaliação de necessidades, seguiuse ao recrutamento para a intervenção. Os bombeiros foram convidados a participar da intervenção ao fim de uma palestra fornecida para todo o agrupamento (em torno de 80 participantes), quando foram abordados sucintamente o fenômeno do stress e as possibilidades de coping individual. Outros 15 bombeiros (que não aqueles participantes da avaliação de necessidades) se ofereceram como voluntários, seis de uma ala e nove de outra ala. Foi estabelecido de comum acordo com os participantes que as sessões seriam feitas no quartel, após o expediente, iniciando 30 minutos após o término da jornada devido à impossibilidade de usar o período 44 de trabalho dada a sobrecarga constante.
Os participantes foram informados sobre o objetivo da intervenção, número e duração das sessões, procedimentos para avaliação e cuidados éticos, e expressaram sua concordância assinando o Termo de Consentimento Livre e Esclarecido. Os 15 voluntários foram distribuídos em duas condições: intervenção 1 e lista de espera. Os seis voluntários da ala Y (nome fictício) foram designados para a intervenção 1 e os nove voluntários da ala Z (nome fictício) ficaram em lista de espera. Após o término da intervenção 1, os voluntários da ala Z foram também submetidos à intervenção, tal como ocorreu com o primeiro grupo. Como ocorreram desistências, participaram do programa até a avaliação final sete bombeiros, sendo três do primeiro grupo (ala Y) e quatro do segundo grupo (ala Z).

\section{Programa de intervenção}

O programa foi feito em 12 sessões. Na primeira e na penúltima sessões foram feitas a aplicação de escalas, a coleta de sangue e a aferição de pressão arterial. A última sessão foi destinada ao fornecimento de feedback, discussão de resultados e encerramento do trabalho. Desse modo, a intervenção foi feita em nove sessões, com duração de 90 minutos cada uma, num total de treze horas e trinta minutos. O conteúdo da intervenção incluiu a discussão de causas e efeitos do stress, fatores de risco e de proteção no curso do desenvolvimento, estabelecimento de metas, enfrentamento focado no problema e na emoção, manejo de tempo, habilidades sociais, solução de problemas, reestruturação de crenças irracionais, relaxamento e prevenção de recaída.

Tipicamente, todas as sessões seguiam uma ordem: (a) recordação e resumo do tema tratado no encontro anterior; (b) introdução ao tema do dia, com perguntas aos participantes acerca de suas concepções sobre o tema abordado; (c) uso de técnicas para aprofundamento no tema, incluindo exposição dialogada (Silva, 2002), relaxamento e visualização (Epstein, 2001), exercícios de automonitoramento, treino de papéis, modelagem e modelação (Gorayeb \& Rangé, 1988) e (d) avaliação da sessão por meio da escolha de uma face impressa em folhas de papel que representasse os sentimentos provocados pela sessão do dia. 
Além disso, foi parte da conduta terapêutica estimular o suporte social entre os participantes sempre que oportuno (ex:: pedindo aos integrantes do grupo que fornecessem feedback positivo acerca do desempenho do colega), a auto-observação e o relato das próprias emoções e ouvi-los empaticamente.

\section{Instrumentos}

Os instrumentos usados na avaliação de indicadores verbais foram:

Roteiro de entrevista não estruturada para levantamento de necessidades: Foi elaborado com o objetivo de identificar as necessidades a serem contempladas na intervenção. Contemplava os temas (a) as fontes de stress no trabalho, (b) o impacto percebido dos estressores ocupacionais sobre a saúde e (c) as estratégias de coping usadas para lidar com o stress no trabalho.

Inventário de sintomas de stress para adultos: A versão original desse instrumento é de autoria de Lipp (1998) e seu objetivo é identificar os sintomas de stress em adultos, a natureza do sintoma (física ou psicológica) e a fase de stress em que o respondente se encontra. É composto por três quadros: o primeiro com 15 itens ou sintomas de stress típicos da fase de alerta, a fase inicial do stress (por exemplo, "tensão muscular" e "vontade súbita de iniciar novos projetos"); o segundo quadro com 15 sintomas descritivos das fases de resistência e quase-exaustão, as fases intermediárias do stress (por exemplo, "sensação de desgaste físico constante" e "problemas com a memória") e o terceiro quadro com 23 sintomas da fase de exaustão, a última e mais grave fase do stress (por exemplo, "angústia/ansiedade diária" e "problemas de pele prolongados"). Ao todo, são 53 sintomas de stress, sendo 19 psicológicos e 34 físicos. Apresenta um coeficiente alfa de 0,91. Aos participantes foi solicitado marcar os itens que descreviam os estados e as sensações vividas nas últimas 24 horas (no primeiro quadro), ou última semana (no segundo quadro), ou último mês (no terceiro quadro).

Escala de coping ocupacional: Foi criada originalmente por Latack (1986) e validada para o Brasil por Pinheiro, Tróccoli e Tamayo (2003). Seu objetivo é avaliar estratégias de enfrentamento utilizadas para lidar com problemas no trabalho. A versão brasileira contém 46 itens, agrupados em três fatores: controle (ações para lidar com a fonte geradora de stress e reavaliações cognitivas de tais fontes, como pedir ajuda para lidar com o problema), esquiva (ações ou cognições de evitação da fonte geradora de stress, como tentar não se preocupar com a situação) e manejo de sintomas (ações ou cognições usadas para lidar com os sintomas do stress, como prática de atividade física). Na versão usada neste estudo, o fator manejo de sintomas foi subdivido em dois fatores: manejo de sintoma (ações ou cognições usadas para lidar com os sintomas de stress sem efeitos colaterais potenciais, como relaxamento) e comportamentos aditivos (ações usadas para lidar com os sintomas de stress com efeitos colaterais potenciais, como comer em excesso). O índice de confiabilidade interna da escala está entre 0,77 e 0,81. O participante foi solicitado a responder conforme uma escala de cores, desde amarelo claro (nunca faço isso) até marrom (sempre faço isso).

Questionário de saúde geral de Goldberg: A escala original foi construída para avaliação de saúde mental por Goldberg na década de 1970. A versão adaptada para o Brasil (Pasquali, Gouveia, Andriola, Miranda \& Ramos, 1996) conta com 60 itens agrupados em cinco fatores: stress psíquico (experiências de tensão, irritação, impaciência, cansaço e sobrecarga, que tornam a vida desgastante e infeliz), desejo de morte (desejo de acabar com a própria vida), desconfiança no desempenho (consciência de ser capaz de desempenhar as tarefas diárias de forma satisfatória), distúrbios do sono (tais como insônia e pesadelos) e distúrbios psicossomáticos (problemas de ordem orgânica, como dores de cabeça). Sua consistência interna, medida pelo alfa de Cronbach, é de 0,95. A versão utilizada neste estudo contava com 43 itens extraídos dos fatores distúrbios de sono, stress psíquico, desconfiança no desempenho e distúrbios psicossomáticos. Utilizou-se uma escala numérica de cinco pontos, de 1 (nunca) até 5 (sempre).

\section{Medidas fisiológicas}

Estudos em psiconeuroimunologia comumente utilizam como variáveis dependentes medidas funcionais ou quantitativas de células do sistema imune (O’Leary, 1990). No presente estudo, optou-se por realizar medidas quantitativas de células do sistema 
imune em vez de medidas de funcionamento dessas células, em função do menor custo com procedimentos de mensuração. Amostras de sangue foram coletadas antes e após a intervenção por estagiários do curso de graduação em Biomedicina de uma universidade da cidade, e submetidas à contagem eletrônica por meio do contador eletrônico marca ABX Pentra 60. Foram em seguida submetidas a uma segunda quantificação, feita por um observador humano através de microscópio de ótica comum Nikon Eclipse E200. O número clinicamente normal de células do tipo linfócitos pode variar entre 900 e 3.300, células do tipo neutrófilos podem variar entre 2.295 e 6.500 e células do tipo monócitos podem variar entre 90 e 800 células (Abbas, Lichtman \& Pober, 2003).

A pressão arterial foi aferida antes e após a intervenção por técnicas em enfermagem que utilizaram esfigmomanômetro do tipo oneróide da marca BD e estetoscópio. O primeiro instrumento foi calibrado junto ao INMETRO antes de proceder à aferição. Resultados clinicamente normais, em adultos, para pressão arterial sistólica situam-se abaixo de 120mmHg (pressão ótima) ou abaixo de 130mmHg (pressão normal) e, para pressão arterial diastólica situam-se abaixo de $80 \mathrm{mmHg}$ (pressão ótima) ou abaixo de $85 \mathrm{mmHg}$ (pressão normal). Resultados acima de $140 \mathrm{mmHg}$ para pressão arterial sistólica e acima de $90 \mathrm{mmHg}$ para pressão arterial diastólica são interpretados como indicadores de hipertensão (Sociedade Brasileira de Cardiologia, 2004).

Os dados obtidos na etapa de avaliação de necessidades foram submetidos à análise de conteúdo. A autora da pesquisa e uma psicóloga previamente treinada realizaram de modo independente a categorização e contagem das freqüências. Eventuais discordâncias foram discutidas e sanadas até se obter o sistema final de categorias. Em virtude do tamanho pequeno da amostra nas condições intervenção 1 e lista de espera, tornou-se inviável a comparação entre grupos. Por isso, para a avaliação dos resultados da intervenção, os dados dos três participantes da primeira condição com os dados dos quatro participantes da segunda condição (somente a primeira avaliação inicial) foram agrupados.

Também em função do pequeno tamanho da amostra, o teste Wilcoxon, não paramétrico, foi escolhido para verificar diferenças grupais entre medidas pré e pós-intervenção em sintomas de stress, problemas em saúde geral, coping, pressão arterial e medidas de imunidade. O Statistical Package for the Social Sciences (SPSS), versão 10.0, foi usado para realização de todas as análises quantitativas.

Por ter o presente estudo um caráter clínico, análises de significância clínica foram feitas para identificar se os participantes haviam mudado suas classificações em pressão arterial (nível ótimo, normal, limítrofe e hipertenso) e em imunidade (abaixo do nível normal, normal, acima do nível normal), comparandose as medidas individuais antes e após a intervenção de acordo com parâmetros normativos convencionais em Medicina. Significância clínica, segundo Jacobson, Follette e Revenstorf (1984), refere-se a mudanças relevantes clinicamente, identificadas quando o sujeito deixa de pertencer ao grupo disfuncional e passa a pertencer ao grupo funcional.

\section{Resultados}

\section{Avaliação de necessidades}

Análise de conteúdo acerca das fontes de stress relatadas durante as entrevistas grupais revelou quatro categorias, por ordem de frequência de relatos: fontes de stress relativas à organização do trabalho (42,5\%), fontes de stress relativas às condições de trabalho (38,3\%), fontes de stress relativas às relações interpessoais (12,8\%) e fontes de stress relativas a conflitos trabalho-família (6,4\%). As construções das três primeiras categorias e de suas respectivas subcategorias foram baseadas no sistema de classificação do contexto de produção de bens e serviços proposto por Ferreira e Mendes (2003).

A organização de trabalho foi descrita como estressante em virtude sobretudo da ocorrência de pressão de tempo (ex.: "não dá tempo de terminar a refeição, tem que sair para atender a ocorrência") e controle (ex.:"tem punição para tudo, sem conferir se é verdade ou não"), seguidos de demandas de produtividade (ex:."cobram o tempo de resposta para atender o chamado, mas cobram para andar devagar na viatura"), problemas quanto às regras (ex.:"ao tocar a sirene tem que sair rápido e bem arrumado") e características da tarefa (ex:."imprevisibilidade vivida no caminho entre o quartel e o local do socorro"). 
As condições de trabalho foram relatadas como estressantes no que se refere predominantemente à ausência de suporte organizacional (ex:" "a gente é como escravo, não tem direito a nada. A diferença é que recebe dinheiro"), seguida de problemas quanto ao ambiente físico (ex.:"barulho da sirene incomoda"), à matéria-prima (ex:: "levantamento de peso"), ao desenvolvimento pessoal (ex:." não valorizam a mão-de-obra qualificada que têm"), aos instrumentos (ex:: "motorista não pode usar luva pra fazer assepsia de material; luva descartável é só pra procedimentos com pessoas"), aos equipamentos (ex.: "farda quente") e à remuneração (ex.: "recebemos seis reais para comprar comida para cada 24 horas de trabalho").

As relações sociais no trabalho foram descritas pelos participantes como estressantes na interação entre o grupo de trabalho (ex:: "existe 'deduragem' e 'puxasaquismo' entre os colegas") e também na interação com agentes externos ao grupo de trabalho (ex:: "0 hospital não faz a parte dele"). Fontes de stress relativas a conflitos família-trabalho foram as relatadas com menor frequência (ex:: "O trabalho afeta a vida lá fora 100\%: afeta a vida social e não pode ter filhos").

Por meio da análise de conteúdo das estratégias de enfrentamento mencionadas pelos participantes durante as entrevistas grupais foi possível identificar duas categorias gerais de coping, que correspondem à classificação de Lazarus e Folkman (1984): coping focado na emoção (ações ou pensamentos destinados a remover ou amenizar efeitos emocionais decorrentes da situação estressora) e coping focado no problema (ações ou pensamentos destinados a lidar diretamente com a situação estressora). A maior parte das estratégias de coping relatadas foi do tipo focado na emoção (80\%), incluindo, por ordem, o engajamento em: lazer (ex.: "assistir comédia"), relações afetivo-sexuais (ex:."vou pra casa do meu namorado pra me aliviar, me afastar dos problemas"), esportes (ex.: "jogar futebol"), comportamentos aditivos (ex:."muitos homens bebem e os mais antigos bebem no trabalho"), negação (ex:.: "finjo que não está acontecendo comigo"), dormir (ex.: "dormir e esquecer o mundo"), humor (ex.: "brincar com os colegas"), usufruto das regalias da profissão (ex.: "ir ao cinema ou discoteca de graça") e outros (ex:."ir à igreja").

A categoria coping focado no problema foi relatada com menor frequência (20\%) e compreendeu apenas três subcategorias, por ordem: resolução de problemas (ex:."tentar resolver os problemas entre nós mesmos e dar menos trabalho às chefias"), desligamento emocional no tratamento à vítima (ex.: "evito me emocionar ao atender a vítima") e redirecionamento do foco de atenção (ex.: "me concentro na satisfação que o trabalho me dá").

Após análise de conteúdo de fontes de stress e estratégias de coping usadas, procedeu-se à análise de conteúdo dos problemas de saúde percebidos como decorrentes do trabalho, os quais puderam ser agrupados em seis categorias: comportamentais, ritmos biológicos, gástricos, osteomusculares, cardiovasculares e outros. Foram relatados com maior frequência problemas comportamentais (33,3\%), como desmotivação para o trabalho e depressão, e problemas em ritmos biológicos (26,7\%), incluindo bruxismo e horários desregulados para fome. As demais categorias tiveram uma frequência menor de relatos: problemas gástricos (8,9\%), como úlcera nervosa; problemas osteomusculares (8,9\%), como problemas de coluna; problemas cardiovasculares $(6,7 \%)$, como varizes, e outros $(15,5 \%)$, como alergia.

Análises preliminares do banco de dados foram feitas para identificar valores fora de escala, dados faltosos e dados extremos. Foram identificados três dados faltosos que foram estimados pelo SPSS por meio do procedimento linear trend at point, o qual utiliza as demais informações do banco de dados para estimar o dado faltoso. Dados extremos univariados e multivariados não foram identificados.

Resultados do teste Wilcoxon evidenciaram que as mudanças observadas no pós-teste foram estatisticamente significativas para sintomas de stress (alerta e resistência/quase-exaustão) e saúde geral (stress psíquico, auto-eficácia e somatização), mas não para coping. A Tabela 1 descreve os resultados do teste Wilcoxon (escores-Z e valores de $p$ ), bem como a mediana e a amplitude de cada variável medida no pré e pós-teste.

Os resultados do leucograma evidenciaram um aumento em neutrófilos para quatro dos participantes e diminuição para três deles. Diminuição em linfócitos e monócitos ocorreu em cinco dos participantes e aumento em dois participantes. Do ponto de vista da significância clínica, as análises de imunidade anteriores à intervenção evidenciaram valores em imunidade fora 
dos limites de normalidade clínica para o participante "P1" (número de monócitos superior ao limite máximo desejável) e"P2" (número de neutrófilos inferior ao limite mínimo desejável). As análises em imunidade após a intervenção mostraram que todos os participantes apresentaram a quantidade de neutrófilos, linfócitos e monócitos dentro dos limites mínimo e máximo desejáveis. Esses resultados apontam, portanto, que as mudanças em imunidade observadas em P1 e P2 tiveram significância clínica. Conforme resultados do teste Wilcoxon apresentados na Tabela 1, tais mudanças não tiveram significância estatística.

A comparação dos resultados pré e pós-intervenção em pressão arterial sistólica mostrou um decréscimo em quatro dos participantes, aumento em dois deles e manutenção em um deles. A mesma comparação em pressão arterial diastólica evidenciou que todos os participantes apresentaram um aumento nessa variável. Do ponto de vista da significância clínica, todos os participantes encontravam-se tanto ao início quanto ao final da intervenção com pressão arterial sistólica e diastólica na classificação ótima ou normal (Sociedade Brasileira de Cardiologia, 2004). Desse modo, as mudanças em pressão arterial não tiveram significância clínica. Os resultados do teste Wilcoxon apontaram significância estatística somente para as mudanças observadas em pressão arterial diastólica (Tabela 1).

\section{Discussão}

A avaliação de necessidades indicou a existência de vários estressores ocupacionais ligados principalmente à organização e condições de trabalho, um repertório restrito de coping focado no problema e a presença de comportamentos aditivos como estratégias de coping focadas na emoção e inúmeras queixas de saúde relativas principalmente à depressão e à desmotivação para o trabalho, alterações nos ritmos biológicos e problemas no aparelho digestivo. A variabilidade de estressores ocupacionais identificados, relativos à organização do trabalho, as condições de trabalho e às

Tabela 1. Diferenças em escores grupais pré e pós-intervenção em sintomas de stress, saúde geral, coping, imunidade e pressão arterial e resultados do teste Wilcoxon $(n=7)$.

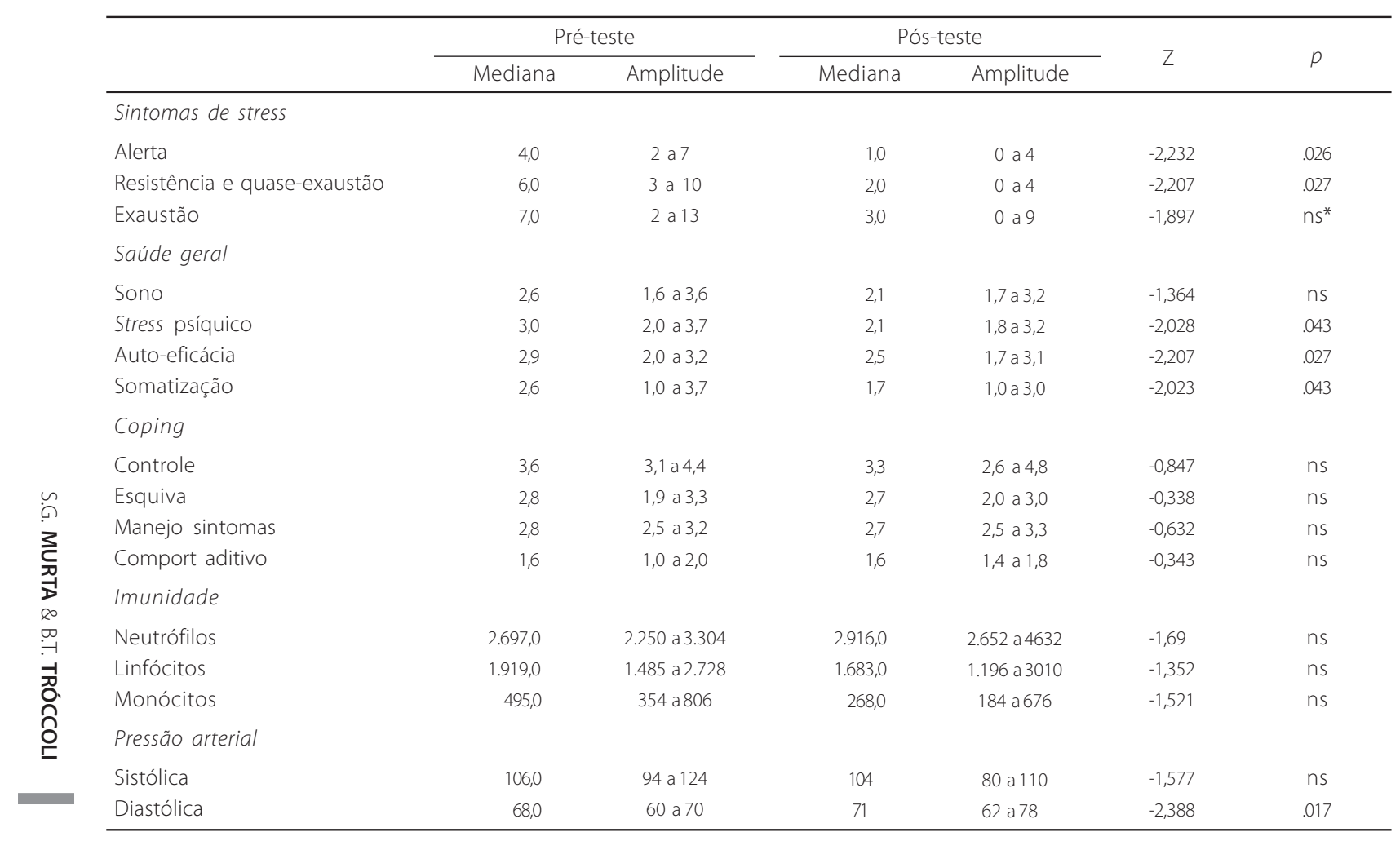

48 Nota*: ns = não significativo, 
relações sociais no trabalho, indicou que um programa de manejo de stress ocupacional focado em mudanças ambientais seria de alta prioridade para a situação. Além dos estressores ocupacionais presentes no ambiente de trabalho, vários bombeiros estavam também trabalhando em outras funções em seus horários de folga, o que resultava em mais de 40 horas de trabalho semanais e alta sobrecarga. Os resultados da avaliação de necessidades foram comunicados às chefias, que já estavam informalmente cientes do grau de stress vivido entre os bombeiros socorristas e motoristas e já estavam analisando possibilidades de mudanças nas condições de trabalho. As chefias receberam os dados da avaliação de necessidades como informações adicionais para fortalecerem seus argumentos junto às instâncias máximas da corporação de bombeiros para que fossem futuramente viabilizadas as mudanças organizacionais necessárias. Enquanto isso, implementou-se o programa de manejo de stress ocupacional focado em coping individual.

O programa foi oferecido para todo o agrupamento de resgate pré-hospitalar (aproximadamente 80 bombeiros), com participação voluntária e fora do horário de trabalho, haja vista a impossibilidade de realizar o programa durante o horário de trabalho, conforme se evidenciou durante a intervenção-piloto. As chefias afirmavam que não havia número de bombeiros suficiente para substituir os bombeiros que se ausentassem para a intervenção e, por outro lado, o trabalho não podia ser interrompido em nenhum momento, por ser um serviço de emergência em saúde.

Apenas 19\% dos bombeiros convidados ( $n=15)$ se ofereceram para participar, e desses apenas $47 \%$ $(n=7)$ permaneceram até $\mathrm{o}$ fim do programa. Acredita-se que um dos fatores responsáveis pela baixa adesão ao programa tenha sido o horário em que foi realizado: o programa ocorreu 30 minutos após o expediente, isto é, após 24 horas de trabalho consecutivas. Apesar de ser esse um momento em que os participantes estariam claramente cansados, foi o horário escolhido por eles próprios, já que muitos não poderiam retornar ao quartel em seu dia de folga por estarem estudando ou trabalhando em outra função ou mesmo descansando. A falta de suporte da organização para que seus integrantes pudessem participar fez com que a amostra fosse altamente seletiva. É certo que aqueles que se ofereceram como voluntários e permaneceram até o pós-teste diferiam em algumas variáveis relevantes do restante da corporação, como no nível de motivação para participação ou no nível de stress ou de crença quanto à eficácia de tratamentos psicológicos. Isso restringe as possibilidades de generalização dos resultados para toda a população de bombeiros socorristas e motoristas dessa corporação de bombeiros.

Quanto aos resultados da intervenção, a comparação dos dados obtidos no pré e pós-teste revelou que houve redução em sintomas de stress e em problemas de saúde geral. Esses resultados estão em acordo com estudos anteriores (Bunce, 1997; Murphy, 1996; van der Klink et al., 2001), os quais também identificaram efeitos positivos de intervenções multimodais em manejo de stress ocupacional sobre respostas individuais de stress.

Todavia não é possível efetivamente estabelecer uma ligação causal entre os dados pós-intervenção e a intervenção, dada a ausência de grupo-controle e seleção aleatória dos participantes. Embora tenha havido uma melhora na condição de sono dos participantes, ela foi estatisticamente não significativa. É possível que a intervenção não tenha sido adequada para combater o impacto da escala noturna de trabalho e os ciclos prolongados de trabalho-descanso sobre a desorganização dos ritmos biológicos ligados aos processos de sono e vigília. O fato de essa intervenção não ter minimizado os problemas de sono, assim como a grande variedade de relatos nas entrevistas grupais acerca de problemas em saúde percebidos como decorrentes do trabalho, sugere novamente a necessidade de intervenções para manejo de stress focadas na organização como estratégia mais indicada para a redução desse tipo de problema.

As estratégias de coping a problemas no trabalho permaneceram inalteradas. Esses resultados podem ter ocorrido em resposta ao conteúdo da intervenção, que não forneceu treinamento em coping específico para estressores comuns na rotina de trabalho. Ao contrário, foi discutido coping a situações diversas da vida, nos múltiplos papéis desempenhados pelos participantes. Uma segunda explicação para esses resultados é que o contexto ocupacional não permitia escolhas aos participantes (van der Klink et al., 2001). Essa hipótese encontra sustentação nos resultados da avaliação de necessidades, que apontaram uma predominância do 
uso de estratégias de coping focadas na emoção, em detrimento de estratégias focadas no problema. Segundo a literatura, estratégias focadas na emoção são usadas quando não há o que fazer para lidar diretamente com a situação estressora (Bond et al., 1984). Esse quadro é bastante similar ao ambiente de trabalho dos bombeiros, caracterizado por diversas e intensas fontes de stress, pouco passíveis de modificação por meio de coping individual.

Os resultados em imunidade e pressão arterial apontaram mudanças clinicamente significativas (em imunidade) e estatisticamente significativas (em pressão arterial). Contudo tais resultados devem ser interpretados com cautela devido ao fato de ter sido feita uma medida única antes e após a intervenção, além do tamanho pequeno da amostra e da ausência de grupo-controle. Conclusões mais sólidas acerca de efeitos benéficos de intervenções multicomponentes para manejo de stress deverão aguardar até que estudos futuros sejam conduzidos, com delineamentos mais favoráveis à validade interna.

Embora tenha sido planejado um estudo quase experimental, contando com uma lista de espera que cumpriria a função de grupo-controle, na prática isso não ocorreu. A intervenção foi implementada tal como planejada, mas se optou na avaliação por agregar os dados dos dois grupos, num delineamento pré-experimental com pré e pós-teste, em função do tamanho pequeno da amostra e da mortalidade experimental, o que impedia a comparação entre os grupos. Uma das possíveis causas da mortalidade experimental foi o alto custo para participar do programa: o horário em que a intervenção foi oferecida era o mesmo horário em que os bombeiros usavam para descansar ou trabalhar em outro local. A experiência da implementação desse programa sugeriu ser fundamental que os custos para participação na intervenção sejam reduzidos de modo a maximizar as chances de adesão, minimizar desistências e alcançar um número maior de pessoas. Isso requer um maior envolvimento da organização com o programa e que ela ofereça suporte necessário.

Considerando as várias limitações deste estudo relativas ao viés da seleção, a mortalidade experimental, a amostra pequena e a ausência de grupo-controle ou medidas repetidas intra-sujeito, torna-se inviável fazer 50 afirmações de causalidade entre a intervenção e a redução de indicadores verbais de saúde no pós-teste, bem como generalizar resultados da avaliação de necessidades e da intervenção para toda a corporação de bombeiros. Novos estudos com esse grupo ocupacional, com amostras maiores, são necessários para identificar em que extensão esses resultados são comuns nessa população ou se foram idiossincráticos dessa amostra. Sugere-se que estudos futuros incluam ações para controle de stress focadas na organização, ou ações combinadas, focadas tanto na organização quanto no indivíduo.

É provável que intervenções organizacionais ou intervenções combinadas sejam as mais adequadas para contextos com grande variabilidade de estressores. Estudos futuros poderão comparar efeitos entre essas modalidades de intervenção bem como monitorar o processo de intervenção a fim de identificar preditores de sucesso ou fracasso na implementação desses programas. Por fim, cabe salientar que os resultados da intervenção foram reportados às chefias, oralmente e por escrito, em conformidade com preceitos éticos. Benefícios, limitações da intervenção e implicações dos dados para mudanças em condições de trabalho foram discutidos extensamente em reunião com todos os membros da hierarquia superior da corporação. Assim, é possível que essa devolução de resultados, em particular da avaliação de necessidades, possa fomentar discussões e ações internas à corporação para prevenção dos estressores ocupacionais e promoção de saúde no trabalho desse grupo ocupacional.

\section{Referências}

Abbas, A. K., Lichtman, A. H., \& Pober, J. S. (2003). Imunologia celular e molecular. Rio de Janeiro: Revinter.

Afonso, L., Abade, F. L., Akerman, D., Coelho, C. M. S., Medrado, K. S. Paulino, J. R., \& Pimenta, S. D. C. (2003). Oficinas em dinâmica de grupo na área da saúde. Belo Horizonte: Edições do Campo Social.

Bellarosa, C., \& Chen, P. Y. (1997). The effectiveness and practicality of occupational stress management interventions. A survey of subject matter expert opinions. Journal of Occupational Health Psychology, 2 (3), 247-262.

Bond, F. W., \& Bunce, D. (2000). Mediators of change in emotion-focused and problem-focused worksite stress management interventions. Journal of Occupational Health Psychology, 5 (1), 156-163.

Bunce, D. (1997). What factors are associated with the outcome of individual-focused worksite stress 
management interventions? Journal of Occupational and Organizational Psychology, 70 (1), 1-17.

Calais, S. L. (2004). Stress pós-traumático: intervenção em vítimas secundárias. In M. E. N. Lipp (Org.), O stress no Brasil: pesquisas avançadas (pp.121-130). Campinas: Papirus.

Cohen, S., \& T. B. Herbert (1996). Health psychology: psychological factors and physical disease from the perspective of human psychoneuroimmunology. Annual Review of Psychology, 47, 113-142.

Corneil, W. (1995). Traumatic stress and organizational strain in the Fire Service. In L. R. Murphy, J. J. Hurrell Jr., S. L. Sauter \& C. P. Keita (Orgs.), Job stress interventions (pp.185-198). Washington, DC: American Psychological Association.

Epstein, G. (2001). Imagens que curam. Campinas: Livro Pleno.

Ferreira, M. C., \& Mendes, A. M. (2003). Trabalho e riscos de adoecimento: o caso dos auditores-fiscais da previdência social brasileira. Brasília: L P \& A.

Gorayeb, R., \& Rangé, B. (1988). Metodologia clínica: técnicas comportamentais. In H. W. Lettner \& B. P. Rangé (Orgs.), Manual de psicoterapia comportamental (pp.73-84). São Paulo: Manole.

Harris, M. B., Baloglu, M., \& Stacks, J. R. (2002). Mental health of trauma-exposed firefighters and critical incident stress debriefing. Journal of Loss and Trauma, 7, 223-238.

Haslam, C., \& Mallon, K. (2003). A preliminary investigation of post-traumatic stress symptoms among firefighters. Work \& Stress, 17 (3), 277-285.

Hawe, P., Degeling, D., \& Hall, J. (1990). Evaluating health promotion: a health worker's guide. Sydney: MacLennan \& Petty.

Jacobson, N. S., Follete, W. C., \& Revenstorf, D. (1984). Psychotherapy outcome research: Methods for reporting variability and evaluating clinical significance. Behavior Therapy, 15, 336-352.

Kiecolt-Glaser, J. (1999). Stress, personal relationships, and immune function: health implications. Brain, Behavior and Immunity, 13, 61-72.

Latack, C. J. (1986). Coping with job stress: measures and future directions for scale development. Journal of Applied Psychology, 71 (3), 377-385.

Lazarus, R. S., \& Folkman, S. (1984). Stress, appraisal and coping. New York: Springer Publishing Company.
Lipp, M. E. N. (1998). Inventário de Sintomas de Stress para Adultos (ISSL). São Paulo: Casa do Psicólogo.

Murphy, R. L. (1996). Stress management in work settings: a critical review of the health effects. Stress Management, $11(2), 112-135$.

Newman, J. E., \& Beeher, T. A. (1979). Personal and organizational strategies for handling job stress: a review of research and opinion. Personnel Psychology, 32, 1-43.

O'Leary, A (1990). Stress, emotion, and human immune function. Psychological Bulletin, 108 (3), 363-382.

Outtlinger, J. M. (1998). Stress and firefighters: An exploratory study. Dissertation- Abstract-International, 58 (8-B), 4503, S: 0419-4217.

Pasquali, L., Gouveia, V. V., Andriola, W. B., Miranda, F. J., \& Ramos, A L. M. (1996). QSG. Questionário de Saúde Geral de Goldberg (adaptação brasileira). São Paulo: Casa do Psicólogo.

Pinheiro, F. A, Tróccoli, B. T., \& Tamayo, M. R. (2003). Mensuração de coping no ambiente ocupacional. Psicologia: Teoria e Pesquisa, 19 (2), 153-158.

Regehr,C., Hill, J., Knott, T., \& Sault, B. (2003). Social support, self-efficacy and trauma in new recruits and experienced firefighters. Stress and Health, 19, 189-193.

Salovey, P., Rothman, A. J., Detweiler, J. B., \& Steward, W. T. (2000). Emotional states and physical health. American Psychologist, 55 (1), 110-121.

Silva, R. C. (2002). Metodologias participativas para trabalhos de promoção de saúde e cidadania. São Paulo: Vetor.

Sociedade Brasileira de Cardiologia. (2004). IV Diretrizes Brasileiras de Hipertensão Arterial. Arquivos Brasileiros de Cardiologia, 82 (Suppl. 4), 1-14.

Van der Hek, H., \& Plomp, H. N. (1997). Occupational stress management programmes: a practical overview of published effect studies. Occupational Medicine, 47 (3), 133-141.

Van der Klink, J. J. L., Blonk, R. W. B., Schene, A. H., \& van Dijk, J. H. (2001). The benefits of interventions for work-related stress. American Journal of Health Promotion, 91 (2), 270-276.

Recebido em: 14/12/2005

Versão final reapresentada em: 22/6/2006

Aprovado em: 4/7/2006 
